\title{
Patient motivation in the management of diabetes
}

\author{
Dharshini Karuppiah ${ }^{1}$, Muditha Weerakkody ${ }^{2}$ \\ ${ }^{1}$ Diabetes and Endocrinology unit, Teaching Hospital, Batticalloa
}

${ }^{2}$ Diabetes and Endocrinology unit, Teaching Hospital, Karapitiya

Looking at the alarming statistics, South Asians have a disproportionately higher incidence of diabetes and compared to the general population and the disease develops 5 to 10 years earlier. This is a profoundly worrying prospect for a disease that shortens lifespan by 5 to 15 years; a leading cause for vision loss, renal failures, heart attacks, strokes and non-traumatic limb amputations. Despite clear evidence that managing diabetes with dietary changes, regular exercise, and adherence to appropriate medications leads to a $53-63 \%$ reduction in complications and a $46 \%$ reduction in mortality, diabetes management and control remain poor in South Asian patients (1). Moreover, the introduction of nearly 40 drugs and drug combinations, including several types of insulin, the proportion of diabetics achieving adequate blood-sugar control remains low.

The complexity of the diabetes care to maintain health and quality of life is a key reason for this failure. Since patients with diabetes should handle majority of their day-to-day care, it is necessary to promote self-management. Researches indicate there are seven essential self-care behaviours in people with diabetes which predict good outcomes. Those are healthy eating, being physically active, monitoring of blood sugar, compliant with medications, good problemsolving skills, healthy coping skills and risk-reduction behaviours. All these seven behaviours have been found to be positively correlated with good glycaemic control, reduction of complications and improvement in quality of life (2).

Though multiple demographic, socio-economic and social support factors can be considered as positive contributors in facilitating self-care activities in diabetic patients, role of clinicians in promoting self-care is vital and has to be emphasized.

The biggest challenge for us as health care providers to promote self-care is dealing with non-adherence. Although there are many reasons for non-adherence, poor attitude towards

the disease, complexity of the drug regimens, cost, and negative patient perceptions about the efficacy of treatment are the crucial ones.

Studies show that South Asians are less likely to exercise or follow a healthy diet. Among South Asian patients with diabetes prescribed oral hypoglycemic agents, ACE inhibitor and statin therapy, only $40-45 \%$ were adherent to these medications, majority $(55 \%)$ of South Asian patients were above recommended A1C targets, 36\% were above blood pressure targets and 58\% were above lipid level targets for diabetes (1).

Patients in our country view self-efficacy negatively; generally appreciate and rely on physicians to provide diabetes advice, not prefer self-management or autonomy. Majority of patients also find it challenging to disclose to physicians if they failed to comply with physician recommendations. Compared to western countries where allied healthcare workers spending more time with patients and have better understanding and concerns, our patients have limited time with the physician.

Among our patients some communities have difference in body image perception where a larger body size is considered more prosperous and healthy. There are misconceptions on benefits of exercise, many elderly think they should just rest. Females experience numerous barriers to engage in regular exercise outside of home, such as safety, wearing appropriate cloths for exercise, and negative attitude that exercising outside is culturally inappropriate.

Adopting a diabetic diet is another challenge for many of our patients. In our society there is a considerable social pressure to eat during social events and family gatherings, as food plays a significant role in maintaining social relationship. Also our traditional diet often contain high quantity of carbohydrate and saturated fat with low protein.

As healthcare providers we should actively involve in developing self-care regimens for each individual patient. Before making recommendations, perceived patient barriers to self-care behaviours must be evaluated. These modifications should be specific for each patient and should be altered depending on the patient's response. The best possible regimen which is practical and realistic to the patient should be prescribed, so that he or she can follow it.

Other important step at the primary care level will be implementing good diabetes self-management education programs with emphasis on motivating good self-care behaviours especially lifestyle modification (3). But the success is on sustaining the healthy behaviour which definitely need periodic reinforcement, adequate social support systems such as allied health care workers, support groups, etc. The system which focus on improving communication; discussing common misperceptions in our community on diabetes management and leveraging cultural beliefs and family as a resource may help to improve diabetes control. 
1. Tanveer Sohal, Parmjit Sohal, Kathryn M. King-Shier, Nadia A. Khan. Barriers and facilitators for Type-2 diabetes management in South Asians: A Systematic Review. Plos one. September 18, 2015.| DOI:10.1371/journal.pone.0136202

2. Shrivastava et al. Role of self-care in management of diabetes mellitus Journal of Diabetes \& Metabolic Disorders 2013, $12: 14$ Page 4 of 5. http://www.jdmdonline.com/content/12/1/14

3. Contreras F, Sanchez M, Martinez MS, Castillo MC, Mindiola A, et al. (2017) Management and education of patient with Diabetes Mellitus. Med Clin Rev. Vol. 3 No. 2:7. 